\title{
Gurvits Syndrome as a Manifestation of Diabetic Ketoacidosis: A Case Report
}

\author{
Gomes Porras M, García Moreno RM* and Melián EM \\ Department of Endocrinology, Hospital Universitario La Paz, Spain
}

Submission: February 08, 2021; Published: March 29, 2021

*Corresponding author: Rosa M García Moreno, Department of Endocrinology, Hospital Universitario La Paz, Paseo de la Castellana 261, CP 28046, Madrid, Spain

\section{Abstract}

Background: Gurvits syndrome is an uncommon and severe form of acute esophagitis characterized by esophageal necrosis that usually affects the lower third of the esophagus and is associated with high morbidity and mortality rates (31.8\%-50\%).

Case report: A 62-year-old patient with long-standing type 2 diabetes mellitus and poor glycemic control presented diabetic ketoacidosis, hemodynamic instability, and melena. An esophagogastroduodenoscopy showed a black esophagus. The patient was successfully treated with conservative management with fluids, insulin, high-dose proton pump inhibitors, and total parenteral nutrition. He showed an improvement in esophageal damage and the diabetic ketoacidosis episode was resolved.

Conclusion: Gurvits syndrome is a rare, multifactorial manifestation of critical illness. It should be suspected in patients with hyperglycemic crises associated with upper gastrointestinal bleeding, especially in advanced age. Treatment should be directed towards the underlying disease and, if applied successfully, patients will have a very high recovery rate.

Keywords: Gurvits syndrome; Acute esophageal necrosis; Black esophagus; Diabetic ketoacidosis; Upper gastrointestinal bleeding

Abbreviations: DKA: Diabetic Ketoacidosis; DM: Diabetes Mellitus; AEN: Acute Esophageal Necrosis; UGB: Upper Gastrointestinal Bleeding; EGD: Esophagogastroduodenoscopy; PPIs: Proton Pump Inhibitors; TPN: Total Parenteral Nutrition; HHS: Hyperosmolar Hyperglycemic Syndrome; RBC: Red Blood Cell

\section{Background}

Diabetic ketoacidosis (DKA) is a life-threatening emergency associated with both type 1 and type 2 diabetes mellitus (DM), although it is more common in type 1 diabetes. This complication can be the initial presentation of undiagnosed type 1 DM or may occur as a consequence of a precipitating cause, such as poor adherence to treatment, infections, or non-infectious diseases. Physiopathology is based on a state of insulinopenia and increased concentration of counter-regulatory hormones that result in hyperglycemia, increase production of ketone bodies, and metabolic acidosis. These mechanisms lead to an inflammatory state with increased oxidative stress, cellular damage, and endothelial dysfunction [1].

The classical presentation of DKA is the triad of polyuria, polydipsia, and weight loss. Gastrointestinal symptoms such as diffuse abdominal pain, nausea, and vomiting are also common. However, other gastrointestinal conditions that are highly uncommon may develop as a result of DKA. Gurvits syndrome, also known as acute esophageal necrosis syndrome (AEN), necrotizing esophagitis, or black esophagus, is a severe form of acute esophagitis, generally characterized by circumferential and diffuse esophageal necrosis that mainly affects the lower third due to its reduced vascularity. The diagnosis is based on endoscopic images of the black esophagus, histologic findings of necrosis of the mucosa, the submucosa and, occasionally, of the muscular layers, as well as acute clinical presentation and exclusion of other causes of the black esophagus, such as recent caustic ingestion, carbon black exposure, pseudo melanosis, melanosis, melanoma, and acanthosis nigricans [2-4].

It is more common in men (male to female ratio of 4:1) with a maximum incidence in the sixth decade of life [2]. The most frequent clinical presentation is upper gastrointestinal bleeding (UGB), usually preceded by gastroesophageal reflux symptoms and 
hemodynamic instability [2]. Prognosis may change and depends more on the patient's age and concomitant conditions than on the course of the esophageal lesions [5]. Complications may include bacterial infection, esophageal stenosis, and gastrointestinal perforation with the risk of mediastinitis and abscess formation. In some cases, the Gurvits syndrome has a fatal course, presenting a reported global mortality rate of $31.8 \%-50 \%[6,7]$.

\section{Case Report}

A 62-year-old Spanish man with a medical history of smoking 10 cigarettes per day, beer consumption (30-50 g of alcohol per day) type $2 \mathrm{DM}$ with 16 years of duration, and poor glycemic control (HbA1c 9.4\%), in treatment with metformin and basal-bolus insulin regimen. He had frequent DKA episodes secondary to poor compliance and dietary transgressions. Regarding microvascular diabetes complications, he presented mixed polyneuropathy and no data of diabetic nephropathy or retinopathy. On the other hand, he had not developed macrovascular complications. His previous medical history also included hypertension, dyslipidemia, proteincalorie malnutrition of moderate degree (BMI 19.5), vitamin D deficiency, depression, mild-to-moderate chronic hypo-osmolar hyponatremia of multifactorial etiology (medications, alcohol, low dietary sodium intake), chronic alcohol-related liver disease with Child-Pugh grade A6, Wernicke encephalopathy, chronic pancreatitis, and complicated peptic ulcer that had required sclerotherapy.

In May 2018, two months after a routine check-up with his usual endocrinologist, he presented to the Emergency Department with a new episode of DKA associated with melena, without hemodynamic instability. He underwent an esophagogastroduodenoscopy (EGD), which showed that the esophageal mucosa was covered by black fibrin from the dental arcade to the cardia (black esophagus), as well as three superficial duodenal ulcers without active bleeding covered by fibrin located in the first portion of the duodenum. No biopsies were taken due to perforation risk. Conservative management with fluids, insulin, high-dose proton pump inhibitors (PPIs), and total parenteral nutrition (TPN) was decided. Five days later, a subsequent EGD was performed and showed denudation of esophageal mucosa throughout the entire length of the esophagus, along with whitish and reddish patches. No bleeding was detected. He did not develop complications or sequelae and was discharged eight days after admission. Currently, he continues his follow-up in our hospital and has not presented any more UGB or DKA episodes.

\section{Discussion}

Gurvits syndrome is uncommon. Two large retrospective series reviewed the results of more than 100,000 endoscopies and the estimated incidence was $0.01 \%$ (12 patients from both series) $[5,8]$. Another retrospective analysis involving 10,295 endoscopy studies showed an incidence of $0.28 \%$ (29 patients) [9]. For a long time, this condition was observed only in post-mortem examinations, until 1990 when Goldenberg et al reported the first endoscopic description [10]. In 2007 Gurvits et al classified it as a clinical syndrome [2]. The physiopathology is multifactorial, resulting from a combination of tissue hypoperfusion, damage of local protective barriers, and gastric outlet obstruction with chemical damage from acid reflux [11].

The predisposing conditions are advanced age, male gender, malnutrition, immunosuppression, alcoholism, valvopathy associated with DM, arteriosclerosis, cardiomyopathy, nephropathy, thromboembolic events and coagulopathy, gastric volvulus, hemodynamic instability, and sepsis. All this induces tissue hypoperfusion, systemic inflammatory response syndrome associated with severe acute pancreatitis, DKA or lactic acidosis, bleeding, hypothermia, thoracic aortic injury, and shock, all of which lead to esophageal ischemic compromise.

Acute decompensated diabetes may cause esophageal hypoperfusion as a result of osmotic diuresis-induced hypovolemia and diabetic microangiopathy. Apart from this, poor metabolic control is associated with an alteration of the intrinsic barrier of the distal esophagus, decreased esophageal motility, and delayed gastric emptying. Yasuda et al reported 29 admissions between 2002 and 2004 and observed that patients with symptoms compatible with UGB (7 patients) underwent EGD. Of these, four patients $(57 \%)$ were diagnosed with AEN and the other three showed normal findings in the endoscopy [12].

Therefore, clinical suspicion of Gurvits syndrome in patients with DKA or hyperosmolar hyperglycemic syndrome (HHS) and UGB is essential for diagnosis. Malnutrition is another risk factor involved in Gurvits syndrome development due to impaired wound healing and the esophageal mucosal defense system being compromised. There is no effective specific treatment, but life support measures such as antibiotic therapy and insulin are recommended to correct the underlying medical conditions that lead to hypoperfusion. Other necessary treatments include fluid therapy to restore hemodynamic, acid-base, and metabolic imbalance; high-dose PPIs; nutritional support, usually with parenteral nutrition; and red blood cell (RBC) transfusions to treat severe anemia. In the case of complications, the treatment goes from esophageal dilations to the surgical approach involving esophagectomy.

\section{Conclusion}

Gurvits syndrome is a potential but rare complication of DKA. It should be considered in the differential diagnosis in patients with UGB-associated DKA, especially in the elderly. Early diagnosis of this condition is crucial due to the high risk of esophageal perforation and mortality. The main challenge is to diagnose the etiology of the AEN as the prognosis will depend on it. The therapeutic approach should be multidisciplinary and aimed at restoring hemodynamic, acid-basic, metabolic, and acid-peptic balance. Early management of the underlying disease and its serious complications is crucial due to the life risk involved. 


\section{References}

1. Fayfman M, Pasquel FJ, Umpierrez GE (2017) Management of Hyperglycemic Crises: Diabetic Ketoacidosis and Hyperglycemic Hyperosmolar State. Med Clin North Am 101(3): 587606.

2. Gurvits GE, Shapsis A, Lau N, Gualtieri N, Robilotti JG (2007) Acute esophageal necrosis: a rare syndrome. J Gastroenterol 42(1): 29-38.

3. Dumas O, Barthélémy C, Billard F, Dumollard JM, Boucheron S, et al. (1990) Isolated melanosis of the esophagus: systematic endoscopic diagnosis. Endoscopy 22(2): 94-95.

4. Ertekin C, Alimoglu O, Akyildiz H, Guloglu R, Taviloglu K (2004) The results of caustic ingestions. Hepatogastroenterology 51(59): 13971400 .

5. Lacy BE, Toor A, Bensen SP, Rothstein RI, Maheshwari Y (1999) Acute esophageal necrosis: report of two cases and a review of the literature. Gastrointest Endosc 49(4): 527-532.

6. Gurvits GE (2010) Black esophagus: acute esophageal necrosis syndrome. World J Gastroenterol 16(26): 3219-3225.
7. Kim YH, Choi SY (2007) Black esophagus with concomitant candidiasis developed after diabetic ketoacidosis. World J Gastroenterol 13(42): 5662-5663.

8. Moretó M, Ojembarrena E, Zaballa M, Tánago JG, Ibánez S (1993) Idiopathic acute esophageal necrosis: not necessarily a terminal event. Endoscopy 25(8): 534-538.

9. Augusto F, Fernandes V, Cremers MI, Oliveira AP, Lobato C, et al. (2004) Acute necrotizing esophagitis: a large retrospective case series. Endoscopy 36(5): 411-415.

10. Kondo T (2018) Acute necrotizing esophagitis. Cleve Clin J Med 85(12): 912-913.

11. Ben Soussan E, Savoye G, Hochain P, Hervé S, Antonietti M, et al. (2002) Acute esophageal necrosis: a 1-year prospective study. Gastrointest Endosc 56(2): 213-217.

12. Yasuda H, Yamada M, Endo Y, Inoue K, Yoshiba M (2006) Acute necrotizing esophagitis: role of nonsteroidal anti-inflammatory drugs. J Gastroenterol 41(3): 193-197.

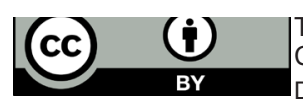

This work is licensed under Creative Commons Attribution 4.0 Licens

DOI: 10.19080/JETR.2020.06.555676 